\title{
ATRIAL WAVES ON ARTERIAL PRESSURE RECORDS IN NORMAL RHYTHM, HEART BLOCK, AND AURICULAR FLUTTER
}

BY

\author{
SHEILA HOWARTH \\ From the Institute of Cardiology, National Heart Hospital \\ Received October 8, 1953
}

Waves produced by atrial contraction have been frequently noted on arterial tracings since Chauveau and Marey (1863) recorded them in the aorta and carotid artery of the horse. Atrial waves have also been observed on arterial pulse tracings in man: François-Franck (1890) reported them in carotid tracings in aortic insufficiency; Webster (1901) and Mackenzie (1905) recorded

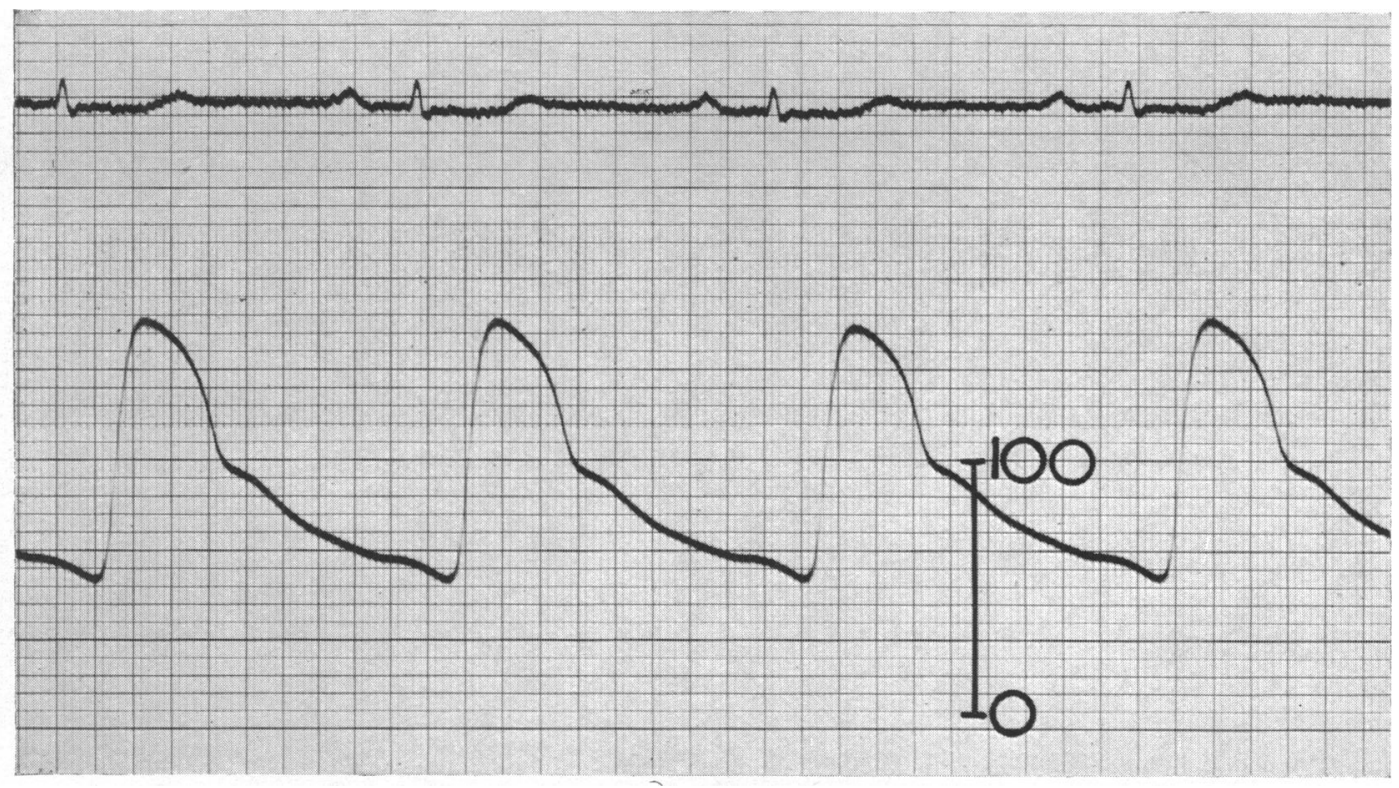

Fig. 1.-Normal rhythm. Atrial waves are seen before the main pulse wave. A prolonged $\mathbf{P}-\mathbf{R}$ interval makes them more apparent, as in this case. Time marker 0.1 and $0.02 \mathrm{sec}$. Calibrations in $\mathrm{mm}$. $\mathrm{Hg}$.

them on radial pulse tracings in heart block; and Draper (1910) drew attention to them on records taken from aortic aneurysms.

Continuous pressure records taken from the brachial artery of patients with sinus rhythm, heart block, and auricular flutter during blood pressure measurement for other purposes have shown pressure waves caused by atrial contraction. Examples are shown in this paper. All records were taken with an intra-arterial needle and capacitance manometer (Hansen, 1949). 


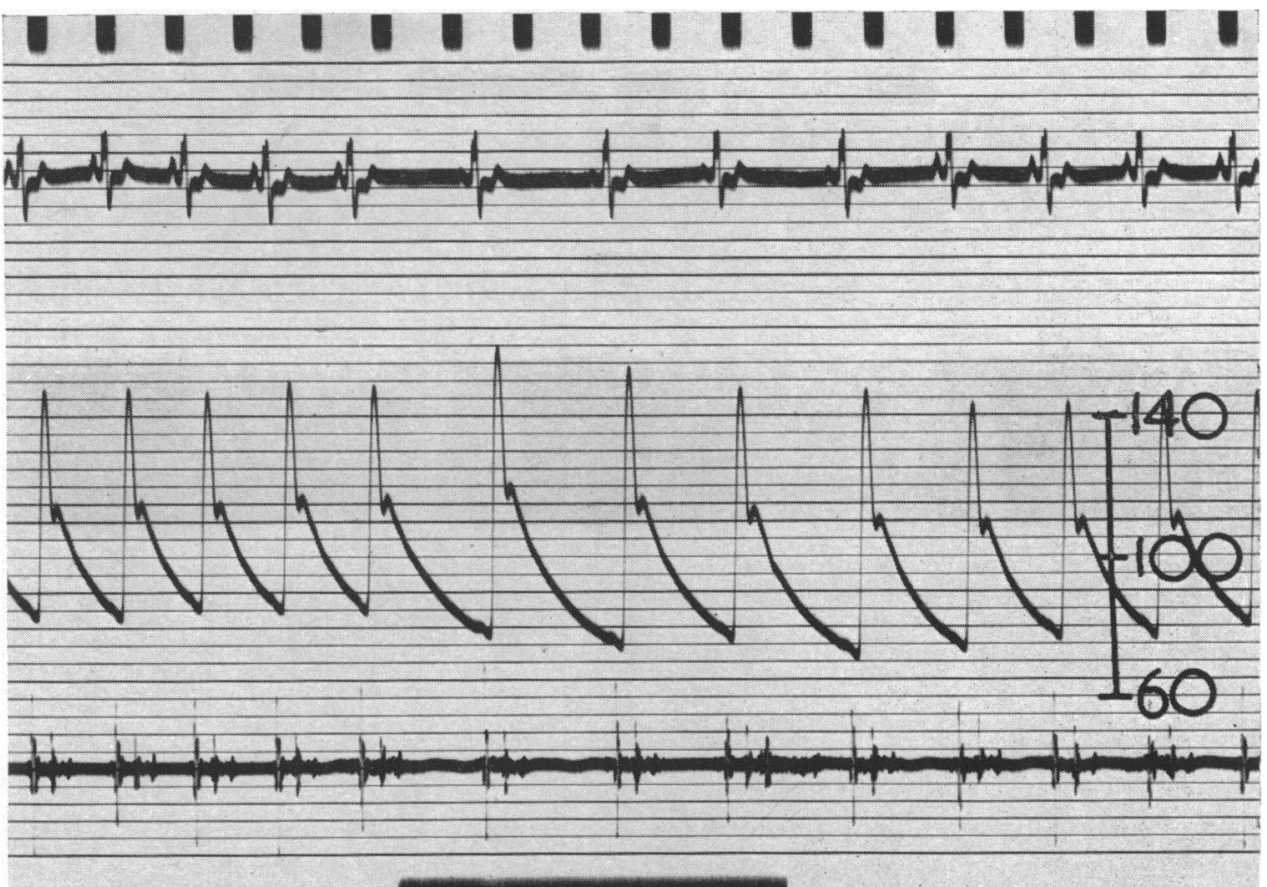

Fig. 2.-Normal rhythm. The atrial waves are well seen when the heart is slowed by carotid sinus pressure (signalled at bottom of tracing). From above downwards: time in seconds, lead II, brachial arterial pressure, and phonocardiogram.

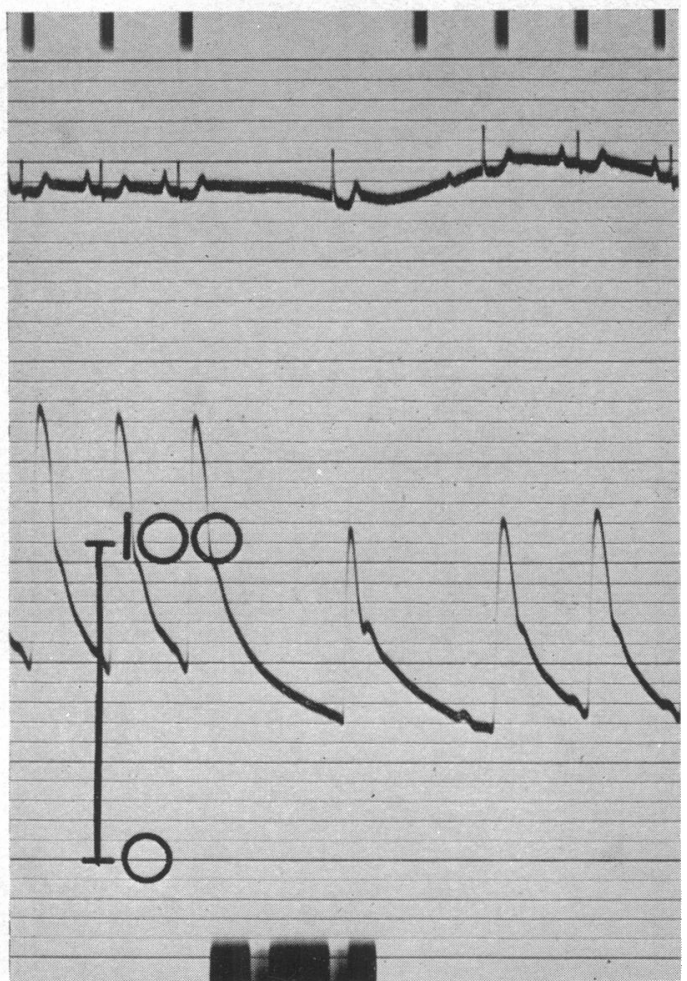
Fig. 3.- Normal rhythm. When atrial activity is in-
hibited by carotid sinus pressure (signalled at bottom), the atrial waves disappear but re-appear with the first $\mathbf{P}$ wave. Time in seconds.

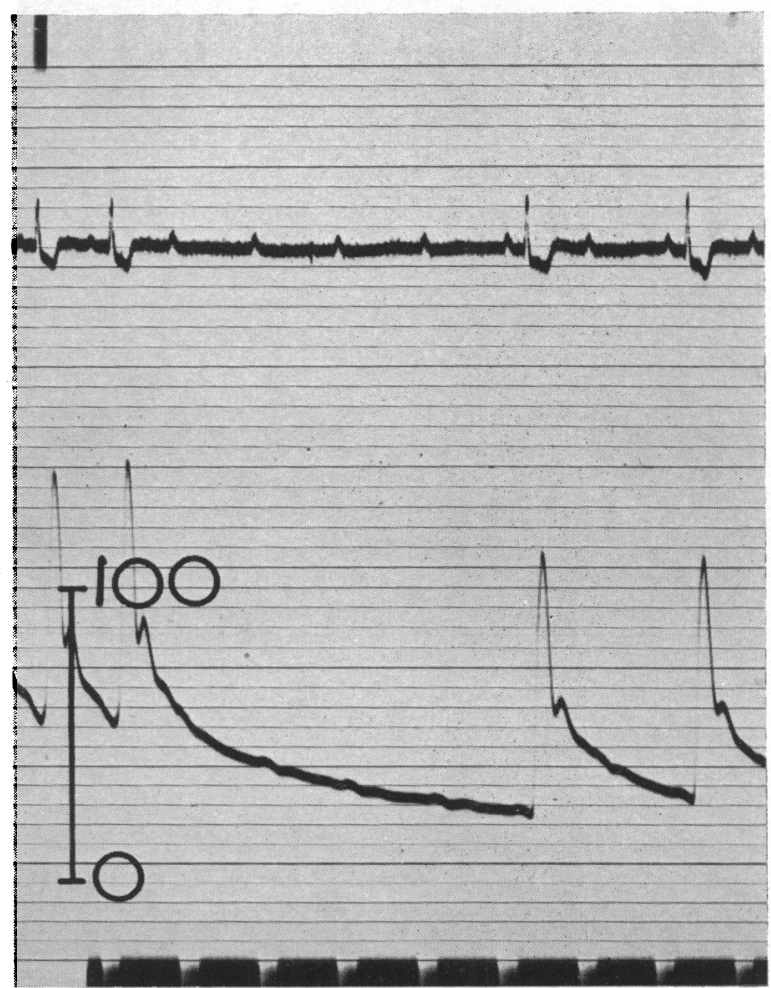

FIG. 4.-Normal rhythm. Carotid sinus pressure (bottom of tracing) inhibits ventricular activity, but atrial contractions continue as shown by $\mathbf{P}$ waves on the electrocardiogram and atrial waves on the brachial artery tracing. Time in seconds. 


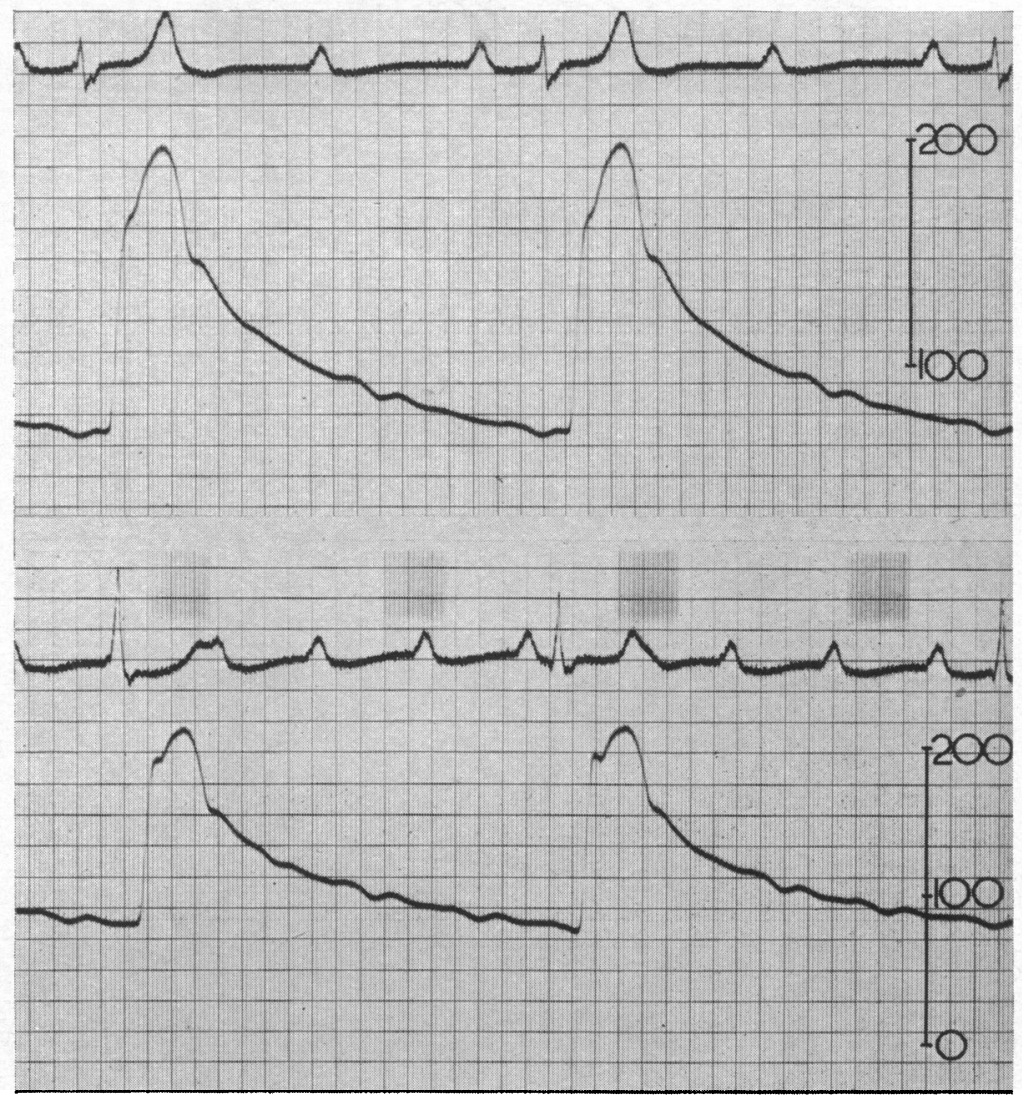

Fig. 5.-Heart block. Atrial waves are seen on two records from the same patient. Above, when in 3:1 block; below, in complete heart block. Time marker 0.1 and $0.02 \mathrm{sec}$.

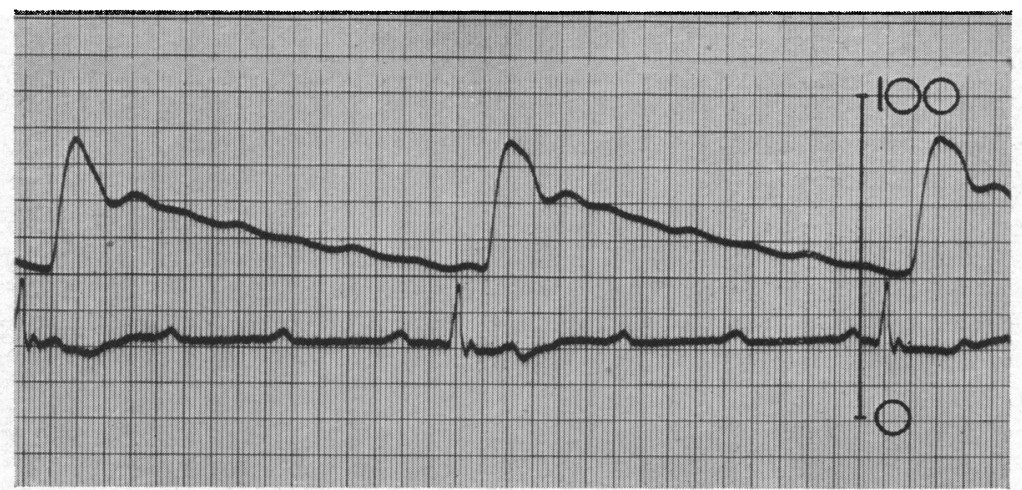

FIG. 6.-Heart block. Atrial waves may occur when the blood pressure is low. This patient developed complete heart block following cardiac infarction. Time marker 0.1 and $0.02 \mathrm{sec}$. 


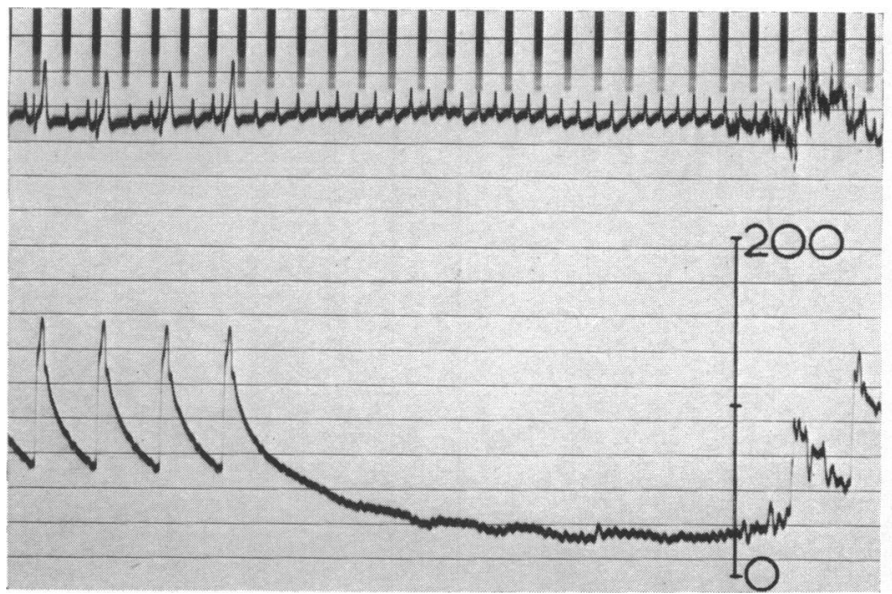

Fig. 7.-Atrial waves are seen on the brachial artery pressure record throughout a Stokes-Adams attack with ventricular asystole. Time in seconds.

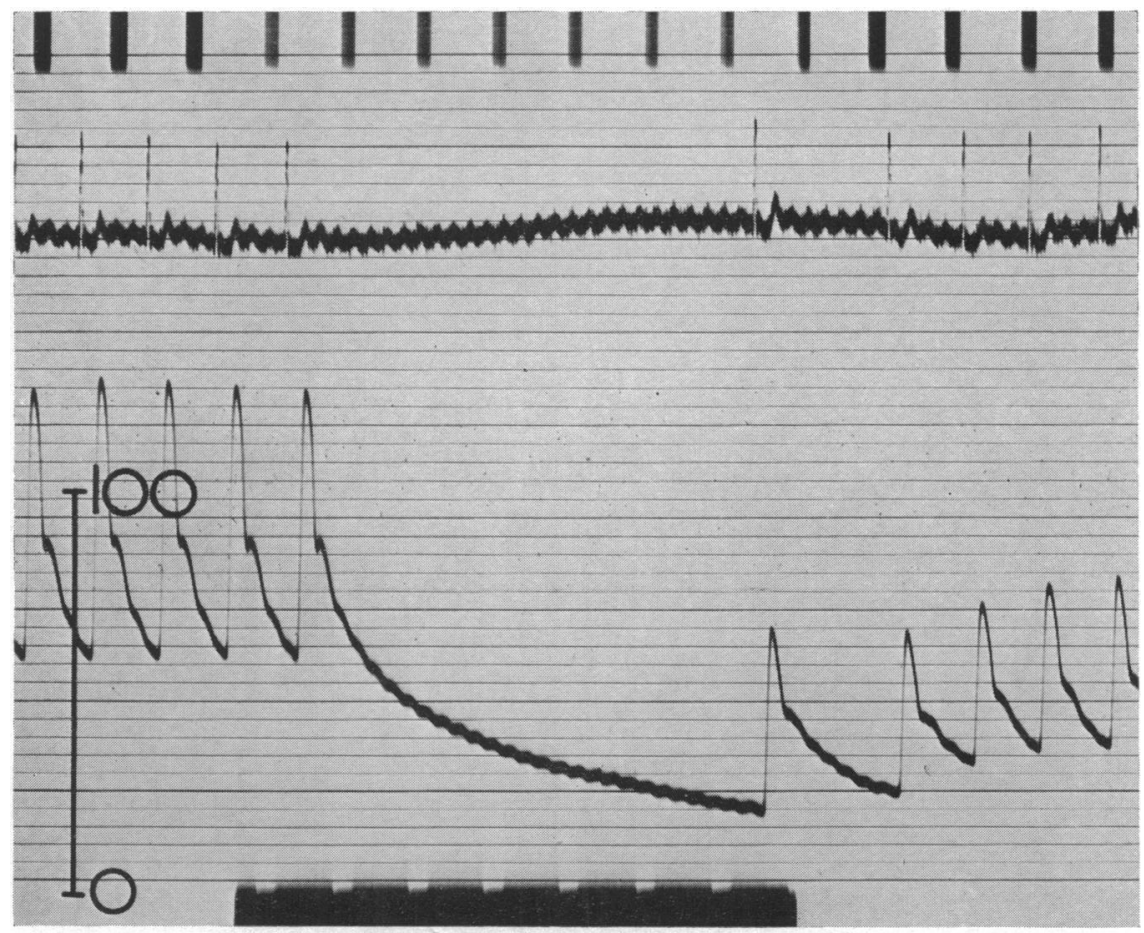

Fig. 8.-Auricular flutter. Carotid sinus pressure (signalled) inhibits ventricular activity, and waves due to atrial contraction are seen on the brachial artery tracing. Time in seconds. 


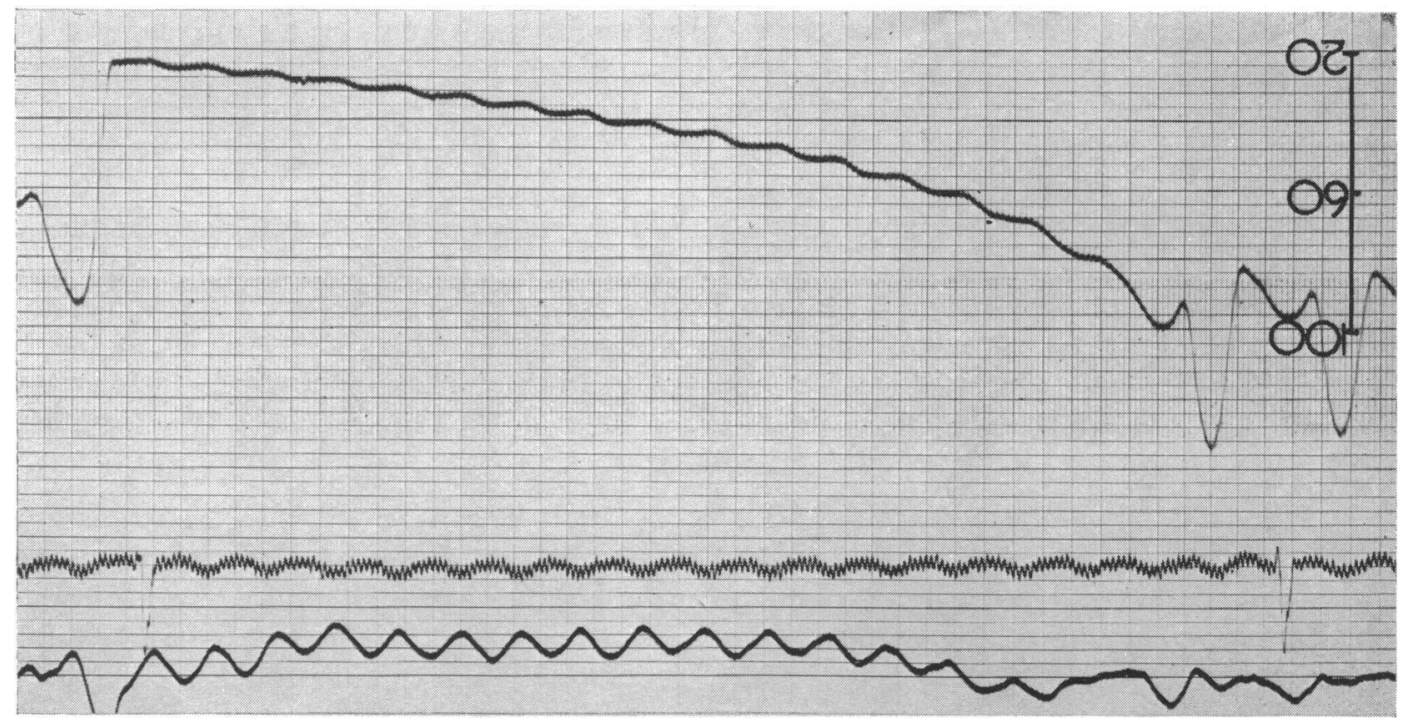

FIG. 9.-Auricular flutter. From above downwards: jugular venous pulse, electrocardiogram lead II, and brachial artery pressure. Time marker 0.1 and $0.02 \mathrm{sec}$. Tracing taken during carotid sinus pressure. The pulse, electrocardiogram, and pressure waves are out of phase, excluding instrumental artefact.

Normal Rhythm. The atrial wave when present occurs immediately before the main deflection of the arterial pulse wave (Fig. 1). Carotid sinus pressure in some cases may help to confirm the atrial origin of the waves. Thus a constant relationship of atrial and ventricular waves to each other and to the electrocardiogram is seen during induced sinus bradycardia (Fig. 2); the atrial wave is abolished when carotid sinus pressure inhibits atrial activity (Fig. 3), but is well demonstrated when ventricular contractions are temporarily arrested (Fig. 4).

The atrial wave when it occurs normally before the main deflection of the arterial pulse consists of a single monophasic positive wave, but when isolated from ventricular events it appears to consist of three deflections, first positive, then negative, then positive again.

Heart Block. Atrial waves are frequently well seen in records from patients with partial or complete heart block (Fig. 5). They may occur with form unchanged over a wide range of arterial pressure levels (Fig. 6). They are also seen during Stokes-Adams attacks when atrial contractions continue during ventricular asystole (Fig. 7), and when the blood pressure may reach the level of 20-25 mm. Hg (Barlow and Howarth, 1953).

The form of the atrial wave in heart block is the same as in subjects with normal rhythm; although the amplitude appears to be larger, it has not been observed to exceed $7 \mathrm{~mm}$. $\mathrm{Hg}$.

Auricular Flutter. In spite of the rapid rate of atrial contractions, atrial waves may appear on the arterial pressure record in some cases of flutter. In $2: 1$ flutter, the rapid ventricular rate obscures atrial events, but in $4: 1$ flutter, atrial waves may be seen on the descending limb of the arterial pressure wave. Carotid sinus pressure by slowing the ventricular rate may demonstrate the atrial waves satisfactorily (Fig. 8).

The positive-negative-positive form of the wave is lost in flutter (Fig. 9), presumably owing to the rapid rate of atrial contraction.

\section{Discussion}

The form of the atrial waves on direct arterial pressure tracings does not differ significantly from that recorded on pulse tracings by the polygraph and sphygmograph in cases with sinus rhythm and heart block. No reference has been found, however, to the occurrence on pulse tracings of atrial waves in auricular flutter. 
It was stated by Webster (1922) that the atrial waves disappeared when the blood pressure fell below a certain level. This may well have been due to his method of recording, since direct intraarterial tracings show that they occur in unchanged form and amplitude over a wide pressure range. The measured amplitude of the waves, however, is such that it appears improbable that they could be detected by the most skilled palpating finger.

The mechanism producing the atrial waves on the arterial pulse has been the subject of much speculation. It is agreed that opening of the aortic valves by atrial systole against the higher aortic diastolic pressure is unlikely. Some have thought that the waves were due to bulging of the aortic valves as the atrial systolic wave was transmitted through the left ventricle (Frank, 1905). Others have considered that the contracting atrium transmitted an extra-arterial impulse to the column of blood in the aorta (Mackenzie, 1914; Webster, 1922; Lewis, 1925; Frank, 1926). The form of the recorded pressure wave would be compatible with either mechanism.

\section{SUMMARY}

Atrial pressure waves are not uncommonly recorded on intra-arterial pressure tracings from the brachial artery. They have been observed in patients with normal rhythm, partial and complete heart block, and auricular flutter. They are also seen during Stokes-Adams attacks.

Carotid sinus pressure may be used to demonstrate the presence of the waves and their atrial origin.

The isolated atrial wave has a positive-negative-positive form, and has not been observed to exceed $7 \mathrm{~mm}$. $\mathrm{Hg}$ in amplitude.

No evidence is available to determine whether the wave results from an extra-arterial impact of the contracting atrium on the aorta, or from a pressure transfer across the aortic valves during atrial systole.

I am grateful to Sir John Parkinson and to Dr. Evan Bedford for furnishing me with excellent polygraph records and books relating to this subject. I am indebted to Mr. J. G. Davies for technical assistance. The cases studied were under the care of the Physicians to the National Heart Hospital.

\section{REFERENCES}

Barlow, E. D., and Howarth, S. (1953). Brit. med. J., 2, 863.

Chauveau, A., and Marey, E. J. (1863). Mém. Acad. Méd., 26, 268.

Draper, G. (1910-11). Heart, $2,84$.

Francois-Franck, M. (1890). Arch. de Physiol., 22, 395.

Frank O. (1905). Zeitsch. Biol., 46, 441.

- (1926). Sitzungsber. Geselsch. Morph. Physiol. in München, 37, 33.

Hansen, A. T. (1949). Pressure Measurement in the Human Organism. Teknisk Forlag, Copenhagen.

Lewis, T. (1925). The Mechanism and Graphic Registration of the Heart Beat. Shaw and Sons, London.

Mackenzie, J. (1905). Brit. med. J., 1, 587.

(1914). Diseases of the Heart. Oxford Med. Publications, Henry Frowde, and Hodder and Stoughton, London.

Webster, A. (1901). Glasgow Hosp. Rep., 3, 413.

(1922). Cardiac Arrhythmia and the Neocardiology. Watts and Co., London. 